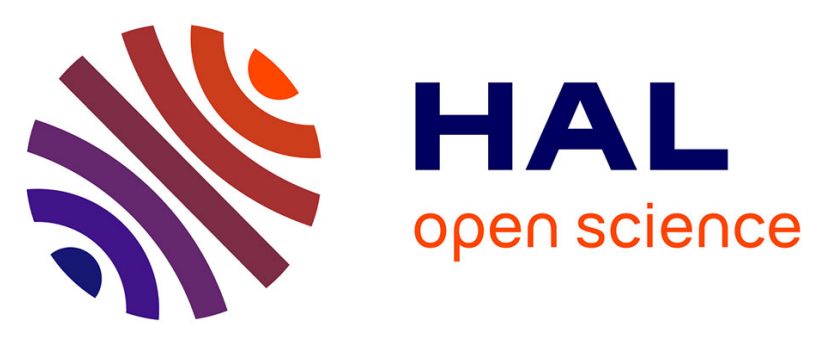

\title{
Discrete-time twisting controller without numerical chattering: analysis and experimental results with an implicit method
}

\author{
Olivier Huber, Vincent Acary, Bernard Brogliato, Franck Plestan
}

\section{- To cite this version:}

Olivier Huber, Vincent Acary, Bernard Brogliato, Franck Plestan. Discrete-time twisting controller without numerical chattering: analysis and experimental results with an implicit method. CDC 2014 - IEEE 53rd Annual Conference on Decision and Control, Dec 2014, Los Angeles, United States. pp.4373-4378, 10.1109/CDC.2014.7040071 . hal-01066689

\section{HAL Id: hal-01066689 \\ https://hal.inria.fr/hal-01066689}

Submitted on 22 Sep 2014

HAL is a multi-disciplinary open access archive for the deposit and dissemination of scientific research documents, whether they are published or not. The documents may come from teaching and research institutions in France or abroad, or from public or private research centers.
L'archive ouverte pluridisciplinaire HAL, est destinée au dépôt et à la diffusion de documents scientifiques de niveau recherche, publiés ou non, émanant des établissements d'enseignement et de recherche français ou étrangers, des laboratoires publics ou privés. 


\title{
Discrete-time twisting controller without numerical chattering: analysis and experimental results with an implicit method
}

\author{
Olivier Huber, Vincent Acary, Bernard Brogliato and Franck Plestan
}

\begin{abstract}
In this note, we present an implementation of the twisting controller on an electropneumatic plant for a tracking control problem. Implicit and explicit discrete-time twisting controllers are considered, and some implementation details are provided. Experimental results are provided and analyzed. They sustain the theoretical superiority of the implicitly discretized version, as shown in previous work. The main advantages of the implicit method are better tracking and drastic reduction in the input and output chattering. This is achieved without modifying the controller structure compared to its continuoustime version.
\end{abstract}

Index Terms - twisting controller, sampled-data system, implicit discretization, electropneumatic actuator

\section{INTRODUCTION}

Implementation of control laws is almost exclusively done using microcontrollers. This implies that the controller is in discrete-time rather than in continuous-time. In sliding mode control, this can induce a degradation of the performance by contributing to the chattering phenomenon. We call this the numerical chattering. An intense activity over the last 30 years was devoted to the reduction of this numerical chattering, mainly for equivalent control based sliding mode control (ECB-SMC). In the early 90's, second order sliding mode control concept was introduced in [1] and sparked the development of a large wealth of literature. One of the first controller of this kind was the twisting controller which features a discontinuous control action w.r.t the sliding variables. However to the best of our knowledge, few discrete-time versions of the twisting controller have been proposed. The replacement of the signum function by a saturation, common trick to reduce the chattering for first order SMC, has no straightforward extension to the twisting algorithm. It is then fair to assume that the explicit discretization was used to get a discrete-time twisting controller, like in [2].

One of the two discretization methods we consider here is the implicit method. It has been used for a long time in the nonsmooth mechanics community, but it was not applied in control theory until very recently [3]-[5]. In particular the implicit discretization of the twisting controller was first studied in [4]. Roughly speaking, the difference between the explicit and implicit methods in our context

The authors acknowledge the support of the ANR grant CHASLIM (ANR-11-BS03-0007).

O. Huber, V. Acary and B. Brogliato are with INRIA Grenoble Rhône-Alpes, BIPOP project-team, 655 avenue de l'Europe, Inovallée, 38334 Saint-Ismier, France; Franck Plestan is with LUNAM Université, Ecole Centrale de Nantes - IRCCyN UMR CNRS 6597, Nantes, France. \{vincent.acary, bernard.brogliato\} @inria.fr, franck.plestandirccyn.ec-nantes.fr, olivier.huber@inria.fr is the following: given a partition $\left\{t_{k}\right\}$ of a time interval, with the explicit discretization, at the time instant $t_{k}$, the argument of the signum function is the value of the sliding variable at $t_{k}$, whereas with the implicit discretization it is the value at $t_{k+1}$. Despite its name and formulation, the implicitly discretized twisting controller is non-anticipative and induces a well-defined behaviour, as we shall see in Section II. Its main features are the drastic reduction of the output chattering and the reduction of the control input chattering, that is the control input is no more of the "bangbang" type. In the discrete-time sliding regime, the control input is also insensitive to an increase of the gain. To simplify the nomenclature, we refer to the discrete-time twisting controller with an implicit (resp. explicit) discretization as the implicit (resp. explicit) twisting controller.

In this note, we present results from an implementation of both explicit and implicit twisting controllers on an electropneumatic plant. The control problem at hand is the tracking of a sinusoidal trajectory for the position of the end of the piston. The analysis of the gathered data supports the theoretical reduction of the chattering [4] and also the claim that the numerical chattering can be the main source of chattering [5]. This highlights the importance of the discretization process which is unfortunately often overlooked.

In the remainder of this section, we introduce the notations. In Section II we briefly recall the twisting controller in continuous-time as well as in discrete-time. The experimental setup is presented in Section III as well as the control scheme. Then the experimental results are analyzed in Section IV.

Notations: The sliding variable is denoted by $\sigma$ and is supposed to be at least twice differentiable. Let $\Sigma:=(\sigma, \dot{\sigma})^{T}$. The control value changes at time instants $t_{k}$, defined as $t_{k}:=t_{0}+k h$ for all $k \in \mathbb{N}$ with $t_{0}, h \in \mathbb{R}_{+}$. The scalar $h>0$ is called the sampling period. Let $\sigma_{k}:=\sigma\left(t_{k}\right)$ and $\dot{\sigma}_{k}:=\dot{\sigma}\left(t_{k}\right)$ for all $k \in \mathbb{N}$. The tilded variants $\widetilde{\sigma}, \dot{\sigma}$ and $\widetilde{\Sigma}$ are used for variables internal to the controller. Let sgn be the classical single-valued signum function: for all $x>0, \operatorname{sgn}(x)=1, \operatorname{sgn}(-x)=-1$ and $\operatorname{sgn}(0)=0$.

Definition 1 (Multivalued signum function). Let $x \in \mathbb{R}$. The multivalued signum function $\mathrm{Sgn}: \mathbb{R} \rightrightarrows \mathbb{R}$ is defined as:

$$
\operatorname{Sgn}(x)= \begin{cases}1 & x>0 \\ -1 & x<0 \\ {[-1,1]} & x=0 .\end{cases}
$$

If $x \in \mathbb{R}^{n}$, then the multivalued signum function Sgn: $\mathbb{R}^{n} \rightrightarrows$ $\mathbb{R}^{n}$ is defined as: $\operatorname{Sgn}(x):=\left(\operatorname{Sgn}\left(x_{1}\right), \ldots, \operatorname{Sgn}\left(x_{n}\right)\right)^{T}$. 


\section{THE TWISTING CONTROLLER}

\section{A. Continuous-time version}

The twisting algorithm was one of the first second-order sliding mode controllers presented in the literature [1]. It requires the control input $u$ to be of relative degree 2 with respect to the sliding variable $\sigma$, that is

$$
\ddot{\sigma}(x, t)=a(x, t)+b(x, t) u,
$$

with the following bounds: for all $(x, t) \in \mathbb{R}^{n} \times \mathbb{R}_{+}$,

$$
0 \leq K_{m} \leq|b(x, t)| \leq K_{M} \quad \text { and } \quad|a(x, t)| \leq K_{a} .
$$

The control law for the twisting controller is

$$
u \in-r_{1} \operatorname{Sgn}(\sigma)-r_{2} \operatorname{Sgn}(\dot{\sigma}),
$$

and with the conditions

$$
\left\{\begin{array}{l}
\left(r_{1}+r_{2}\right) K_{m}-K_{a}>\left(r_{1}-r_{2}\right) K_{M}+K_{a} \\
\left(r_{1}-r_{2}\right) K_{m}>K_{a},
\end{array}\right.
$$

the closed-loop system (1) and (2) converges to the origin in finite time. Lyapunov functions for this controller have been recently investigated, see [6], [7].

It is worth noting that the controller (2) is by definition multivalued and that the control input is a selection of the closed-loop differential inclusion formed by (1) and (2).

\section{B. Discrete-time version}

The control input computed by a microcontroller is usually a step function, with a value periodically updated. We model the control input function as $\bar{u}=\bar{u}_{k}$ for $t \in\left[t_{k}, t_{k+1}\right)$. When implementing this controller, the task at hand at each time instant $t_{k}$ is to compute the control input value from all the possible values defined by a discretization of (1) and (2). We want to keep the multivalued feature of the controller. This is achieved by using the implicit discretization, which applied on (2) gives

$$
u_{k} \in-r_{1} \operatorname{Sgn}\left(\sigma_{k+1}\right)-r_{2} \operatorname{Sgn}\left(\dot{\sigma}_{k+1}\right),
$$

whereas the explicit discretization yields

$$
u_{k}=-r_{1} \operatorname{sgn}\left(\sigma_{k}\right)-r_{2} \operatorname{sgn}\left(\dot{\sigma}_{k}\right) .
$$

Note that the relation in (4) is not an inclusion since the right-hand side is given at time $t_{k}$. The event where either $\sigma_{k}$ or $\dot{\sigma}_{k}$ is zero is clearly pathological. Hence the signum function is single-valued, contrarily to the continuous-time case. The computation of the control input value is in this case straightforward from equation (4).

With the implicit discretization, a discrete-time version of the dynamics (1) is required to perform this computation. In the following, the discrete-time dynamics of $\Sigma:=(\sigma, \dot{\sigma})^{T}$ is supposed to be affine, that is

$$
\widetilde{\Sigma}_{k+1}=A_{k}^{d} \Sigma_{k}+F_{k}^{d}+B_{k}^{d} \Lambda .
$$

with $\Lambda:=\left(\lambda_{1}, \lambda_{2}\right)$ and $u_{k}=-\left\langle\left(r_{1}, r_{2}\right), \Lambda\right\rangle$, the dependency of $\Lambda$ on $k$ being hidden. At each time instant $t_{k}, \Sigma_{k}=$
$\Sigma\left(t_{k}\right)$ but $\widetilde{\Sigma}_{k+1}$ is in general not equal to $\Sigma\left(t_{k+1}\right)$. If the dynamics (1) is LTI and exact, the discrete-time dynamics obtained using a $\mathrm{ZOH}$ discretization is exact and therefore $\widetilde{\Sigma}_{k+1}=\Sigma\left(t_{k+1}\right)$.

The control input value $u_{k}$ is computed with the solution $\Lambda$ of the following generalized equation

$$
\left\{\begin{array}{l}
\widetilde{\Sigma}_{k+1}=A_{k}^{d} \Sigma_{k}+F_{k}^{d}+B_{k}^{d} \Lambda \\
\Lambda \in-\operatorname{Sgn}\left(\widetilde{\Sigma}_{k+1}\right),
\end{array}\right.
$$

with unknowns $\Lambda$ and $\widetilde{\Sigma}_{k+1}$. Let us analyze this system using tools from convex analysis and variational inequalities. The equivalence $\Lambda \in-\operatorname{Sgn}\left(\widetilde{\Sigma}_{k+1}\right) \Longleftrightarrow \widetilde{\Sigma}_{k+1} \in-\mathcal{N}_{[-1,1]^{2}}(\Lambda)$, with $\mathcal{N}_{[-1,1]^{2}}(\Lambda)$ the normal cone to the square $[-1,1]^{2}=$ $[-1,1] \times[-1,1]$ at the point $\Lambda$, enables us to transform (5) into the generalized equation

$$
0 \in A_{k}^{d} \Sigma_{k}+F_{k}^{d}+B_{k}^{d} \Lambda+\mathcal{N}_{[-1,1]^{2}}(\Lambda) .
$$

More precisely, this inclusion is an equivalent form of an Affine Variational Inequality (AVI) [8]. Solving this AVI consists in finding $\Lambda \in[-1,1]^{2}$ such that for all $w \in$ $[-1,1]^{2}$,

$$
(w-\Lambda)^{T} L_{k}(\Lambda) \geq 0,
$$

with $L_{k}: \Lambda \mapsto A_{k}^{d} \Sigma_{k}+F_{k}^{d}+B_{k}^{d} \Lambda$ an affine map. Let us present some properties of the solution of AVI (6). We use $\operatorname{SOL}\left(L_{k}\right)$ to denote the set of all solutions to the AVI (6).

Lemma 1. The AVI (6) has always a solution.

Proof. Since the mapping $L_{k}$ is continuous and $[-1,1]^{2}$ is a bounded convex set, Corollary 2.2.5, p. 148 in [8] gives the result.

The proposed controller is non-anticipative: a solution to the AVI (6), with a positive semi-definite matrix $B_{k}^{d}$, can be computed using the algorithm proposed in [9] which is implemented in the SICONOS software package $[10]^{1}$. Since the AVI (6) has dimension 2, it is also possible to find the solution by enumeration, that is test the 9 possible cases for $\lambda_{1}$ and $\lambda_{2}$ and pick one that is satisfactory.

\section{EXPERIMENTAL SETUP}

\section{A. Plant dynamics}

Let us now present the physical system, actuators and sensors. The electropneumatic system of the IRCCyN lab (École Centrale de Nantes, France), depicted on Fig. 1, has two actuators. On the left-hand side, there is a double acting electropneumatic actuator (the "main" one) controlled by two servodistributors and composed of two chambers denoted $P$ and $N$. The piston diameter is $80 \mathrm{~mm}$ and the rod diameter is $25 \mathrm{~mm}$. With a source pressure equal to 7 bar, the maximum force developed by the actuator is $2720 \mathrm{~N}$. The air mass flow rates entering the chambers are modulated by two three-way servodistributors. The pneumatic jack horizontally moves a load carriage of mass $M$. This carriage is coupled with the second electropneumatic actuator, the "perturbation" one, on

\footnotetext{
${ }^{1}$ http://siconos.gforge.inria.fr
} 


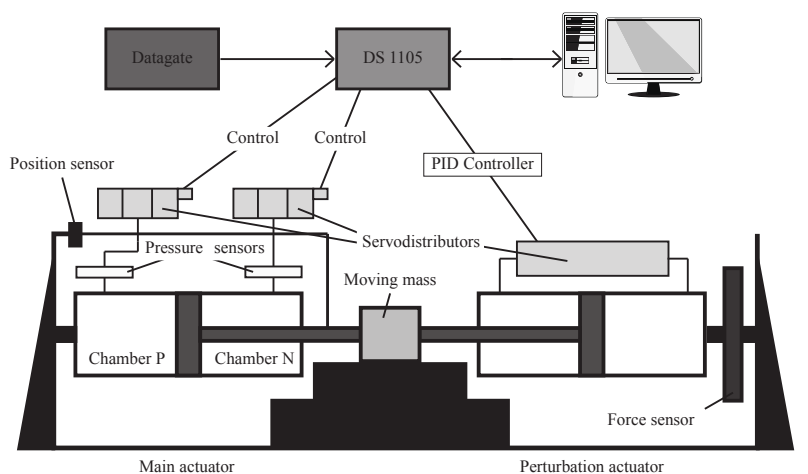

Fig. 1: Scheme of the electropneumatic system

the right-hand side. The goal of the latter is to impress a dynamic load force on the main actuator. This actuator has the same mechanical characteristics as the main one, but the air mass flow rate is modulated by a single five-way servodistributor.

Under some assumptions detailed in [11], the plant dynamics can be written as a nonlinear system affine in the control input $\left[\begin{array}{ll}u_{P} & u_{N}\end{array}\right]^{T}$, with $u_{P}$ (resp. $\left.u_{N}\right)$ the control input of the servo distributor connected to the $P$ (resp. $N$ ) chamber. The model is divided in two parts: the first two equations describe the pressure dynamics in each chamber and the motion of the piston is given by the last two equations. There is a single control objective: make the load position track a reference trajectory. Therefore we set $u:=u_{P}=-u_{N}$, and the dynamics of the electropneumatic experimental setup is

$$
\begin{aligned}
\dot{p}_{P} & =\frac{\kappa r T}{V_{P}(y)}\left[\varphi_{P}+\psi_{P} u-\frac{S}{r T} p_{P} v\right] \\
\dot{p}_{N} & =\frac{\kappa r T}{V_{N}(y)}\left[\varphi_{N}-\psi_{N} u+\frac{S}{r T} p_{N} v\right] \\
\dot{v} & =\frac{1}{M}\left[S\left(p_{P}-p_{N}\right)-b_{v} v-F\right] \\
\dot{y} & =v,
\end{aligned}
$$

with $p_{P}\left(\right.$ resp. $p_{N}$ ) the pressure in the $P$ (resp. $N$ ) chamber, $y$ and $v$ being the position and velocity of the load. The constant $\kappa$ is the polytropic index, $r$ the ideal gas constant, $T$ the temperature (supposed the same inside and outside the chambers) and $b_{v}$ the viscous friction coefficient. The volumes in each chamber are $V_{P}$ and $V_{N}$, both depending on the actuator position $y$. The constant piston section is $S$. The external force applied by the perturbation actuator is denoted by $F$. Finally, $\varphi_{X}$ and $\psi_{X}(X$ being $P$ or $N)$ are both $5^{\text {th }}$ order polynomial functions versus $p_{X}$ [12], that characterize the mass flow rate $q_{X}$ in the chamber $X$ in the following way

$$
q_{X}=\varphi_{X}\left(p_{X}\right)+\psi_{X}\left(p_{X}, \operatorname{sgn}\left(u_{X}\right)\right) u_{X} .
$$

The sources of uncertainty can be the polytropic index $\kappa$, the mass flow, the temperature $T$, the mass $M$, the viscous friction coefficient $b_{v}$ and the disturbance force $F$. They can be modeled by additive bounded functions added to the nominal part of each parameter. As an example, the mass
$M$ can be viewed as the sum of a nominal part and an uncertain one: $M=: M_{n}+\Delta M$, where $\Delta M$ is a bounded uncertainty and $M_{n}$ the nominal value. The control variable $u$ is constrained to take values between -10 and 10 .

\section{B. Control strategy}

The presence of uncertainties motivates the use of a sliding mode control scheme, well-known for its robustness. A first study was already conducted for equivalent-based sliding mode controller, with a comparison between explicit, implicit and saturation methods [13]. The experiments we present here were carried on with the discrete-time twisting controller presented in Section II. Since we are interested in a tracking problem for the position, we choose $y$ as the output. The desired position of the piston is $y_{d}$ and the position error in the tracking problem is $e:=y-y_{d}$. The choice of output leads to a relative degree 3 . Therefore, to bring the relative degree between the sliding variable and the control input to 2 , we define the sliding variable as

$$
\sigma:=\alpha e+\dot{e} .
$$

Its first and second derivatives are

$$
\dot{\sigma}=\alpha \dot{e}+\ddot{e} \text { and } \ddot{\sigma}=\alpha \ddot{e}+\dddot{y}-\dddot{y}_{d},
$$

where

$$
\dddot{y}=\ddot{v}=\frac{1}{M}\left[S\left(\dot{p}_{P}-\dot{p}_{N}\right)-b_{v} \dot{v}-\dot{F}\right] .
$$

Using the relation in (7), we get

$$
\begin{aligned}
\dddot{y}= & \frac{S \kappa r T}{M}\left(\frac{\varphi_{P}}{V_{P}}-\frac{\varphi_{N}}{V_{N}}\right)-\frac{S^{2} \kappa}{M}\left(p_{P}-p_{N}\right) v \\
& +\frac{b_{v}}{M^{2}}\left(S\left(p_{P}-p_{N}\right)-b_{v} v-F\right)-\frac{\dot{F}}{M} \\
& +\frac{S \kappa r T}{M}\left(\frac{\psi_{P}}{V_{P}}-\frac{\psi_{N}}{V_{N}}\right) u .
\end{aligned}
$$

Let us define the following functions

$$
\begin{aligned}
\Phi:= & \frac{S \kappa r T}{M}\left(\frac{\varphi_{P}}{V_{P}}-\frac{\varphi_{N}}{V_{N}}\right)-\frac{S^{2} \kappa}{M}\left(p_{P}-p_{N}\right) v \\
& +\frac{b_{v}}{M^{2}}\left(S\left(p_{P}-p_{N}\right)-b_{v} v\right)+\alpha \ddot{e}-\dddot{y}_{d}
\end{aligned}
$$

and

$$
\Psi:=\frac{S \kappa r T}{M}\left(\frac{\psi_{P}}{V_{P}}-\frac{\psi_{N}}{V_{N}}\right),
$$

which are the nominal functions associated with the dynamics of the sliding variable

$$
\ddot{\sigma}=\Phi+\Delta \Phi+(\Psi+\Delta \Psi) u .
$$

The terms $\Delta \Phi$ and $\Delta \Psi$ are here to take into account uncertainties, like those introduced by the perturbation actuator: $F$ and $\dot{F}$ in (8). The variables $p_{P}, p_{N}$ and $y$ are measured. The implicit controller is constructed in the following way: the 
control input is discretized using the implicit discretization, that is

$$
u_{k} \in-G\left(\operatorname{Sgn}\left(\sigma_{k+1}\right)+\beta \operatorname{Sgn}\left(\dot{\sigma}_{k+1}\right)\right) .
$$

The dynamics of the sliding variable is discretized as follows: expressing the sliding variable dynamics as a first-order ODE, we get

$$
\dot{\Sigma}=A \Sigma+F+B u,
$$

with $\Sigma=\left(\begin{array}{c}\sigma \\ \dot{\sigma}\end{array}\right), A=\left(\begin{array}{cc}0 & 1 \\ 0 & 0\end{array}\right), B=\left(\begin{array}{cc}0 & 0 \\ G \Psi & \beta G \Psi\end{array}\right)$ and $F=\left(\begin{array}{l}0 \\ \Phi\end{array}\right)$. We discretize $\Phi$ and $\Psi$ using the explicit Euler scheme: we consider that $\Phi(t)=\Phi_{k}:=\Phi\left(t_{k}\right)$ and $\Psi(t)=$ $\Psi_{k}:=\Psi\left(t_{k}\right)$ for $t \in\left[t_{k}, t_{k+1}\right)$. For the last step in the discretization of (10), we use the $\mathrm{ZOH}$ method, which yields

$$
\widetilde{\Sigma}_{k+1}=A^{*} \Sigma_{k}+F_{k}^{*}+B_{k}^{*} \Lambda
$$

with $A^{*}:=e^{A h}=\left(\begin{array}{cc}1 & h \\ 0 & 1\end{array}\right), B_{k}:=\left(\begin{array}{cc}0 & 0 \\ G \Psi_{k} & \beta G \Psi_{k}\end{array}\right)$ and $B_{k}^{*}:=\int_{t_{k}}^{t_{k+1}} e^{A \tau} B_{k} \mathrm{~d} \tau=\left(\begin{array}{cc}h^{2} G \Psi_{k} / 2 & \beta h^{2} G \Psi_{k} / 2 \\ h G \Psi_{k} & \beta h G \Psi_{k}\end{array}\right)$, $F_{k}:=\left(\begin{array}{c}0 \\ \Phi_{k}\end{array}\right)$ and $F_{k}^{*}:=\int_{t_{k}}^{t_{k+1}} e^{A \tau} F_{k} \mathrm{~d} \tau=\left(\begin{array}{c}h^{2} \Phi_{k} / 2 \\ h \Phi_{k}\end{array}\right)$. We combine (9) and (11) to get

$$
\begin{aligned}
\widetilde{\sigma}_{k+1} & =\sigma_{k}+h \dot{\sigma}_{k}+\frac{h^{2}}{2} \Phi_{k}+\frac{h^{2}}{2} G \Psi_{k}\left[\lambda_{1}+\beta \lambda_{2}\right] \\
\widetilde{\sigma}_{k+1} & =\dot{\sigma}_{k}+h \Phi_{k}+h G \Psi_{k}\left[\lambda_{1}+\beta \lambda_{2}\right] \\
\lambda_{1} & \in-\operatorname{Sgn}\left(\widetilde{\sigma}_{k+1}\right) \\
\lambda_{2} & \in-\operatorname{Sgn}\left(\widetilde{\dot{\sigma}}_{k+1}\right) .
\end{aligned}
$$

The position $y$ is available but both the speed $v$ and acceleration are computed using a filtered differentiator of the form

$$
D(s)=\frac{s}{1+\tau s}
$$

in the frequency domain.

\section{EXPERIMENTAL RESULTS}

This section is devoted to the analysis of the experimental results obtained on the electropneumatic setup. Recall that the control objective is to make the position of the piston track a sinusoidal trajectory. In the following, the desired trajectory is

$$
y_{d}:=A_{\mathrm{mpl}} \sin (0.2 \pi t) .
$$

The controller was implemented as a Simulink model and then transfered onto a DS1005 dSpace board. We were able to get results with the sampling period $h$ in the range $[3,100] \mathrm{ms}$ and with the gain $G$ in the range $\left[10^{-2}, 10^{7}\right]$. The sliding surface parameter $\alpha$ and the two filtered differentiator time constants require proper tuning for each sampling period. They can drastically alter the performances of the control. Since it appears that both have to be tuned together, preliminary values were obtained using simulations, with a selection based on the average error or precision, and were later tuned on the plant.
We now present results for two criteria: the tracking accuracy and the chattering magnitude on both the input and the output. In each case, we first compare the explicit and implicit methods, before analyzing in more depth the performances of the implicit method.

\section{A. Tracking accuracy}

The tracking error $e=y-y_{d}$ is the quantity we minimize through the twisting controller. Due to the high relative degree of the system, the controller does not bring $e$ to 0 in finite time, but rather $\sigma=\alpha e+\dot{e}$. Once the sliding phase occurs, the convergence of $e$ to 0 is then exponentially fast if $\alpha>0$. The latter parameter controls the speed of convergence: the bigger $\alpha$ is, the faster the error decreases.

To measure the accuracy of the tracking, we compute the average of the absolute value of the error over an interval of 60 s. We call this quantity the precision and we denote it $\bar{e}$. Its analytical formula is

$$
\bar{e}:=\sum_{k=1}^{N} \frac{\left|e\left(t_{k}\right)\right|}{N} \quad \text { with } \quad t_{N}-t_{1}=60 \mathrm{~s} .
$$

On Fig. 2, the precision with both the implicit and explicit

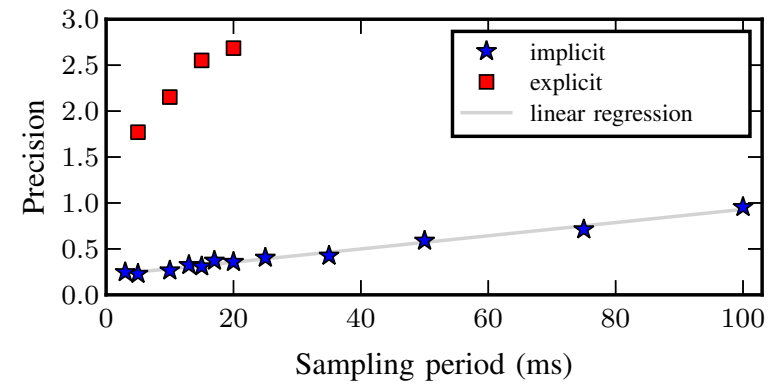

Fig. 2: Evolution of the precision $\bar{e}$ with respect to the sampling time for both implicit and explicit discretizations. The gain used in every capture was $G=10^{5}$.

controllers is displayed for different sampling periods. The implicitly discretized controller clearly yields a better performance than the explicit one, and this for each sampling period where the comparison is possible. Indeed it was not possible to get reliable data for large sampling periods with the explicit controller, since the plant was becoming unstable. The precision appears to increase linearly with $h$, or in other word it is in $\mathcal{O}(h)$. This is underscored by the linear regression plotted in Fig. 2. This may be of surprise since we use a second-order sliding mode controller and the order should be $\mathcal{O}\left(h^{2}\right)$. However, recall that $\sigma=\alpha e+\dot{e}$, with the derivative being computed by a simple filtered differentiator. Hence in (12), the term $\sigma_{k}$ is known with a precision only in $\mathcal{O}(h)$. This problem may be alleviated by the use of another differentiator, like the one proposed in [14].

Let us show more details for a specific sampling period: $h=10 \mathrm{~ms}$. On Fig. $3 \mathrm{a}$ and $3 \mathrm{~b}$, the real and desired trajectories are depicted with, respectively, an implicit (or explicit) controller. On Fig. 3a, the tracking is very accurate: the two curves are undistinguishable. On the other hand, on Fig. $3 b$, the chattering of the real trajectory is visible in the 


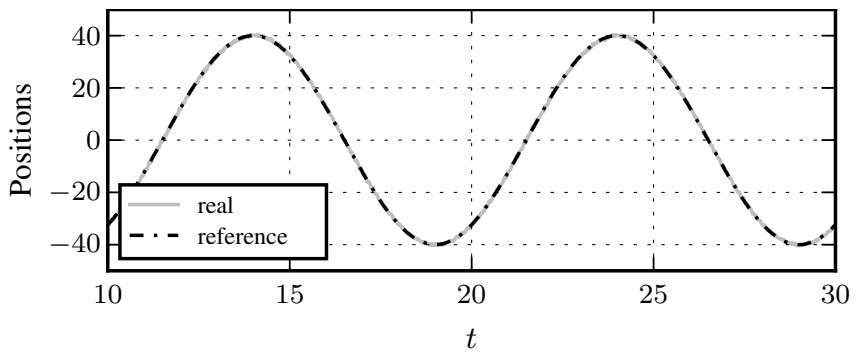

(a) With an implicit discretization.

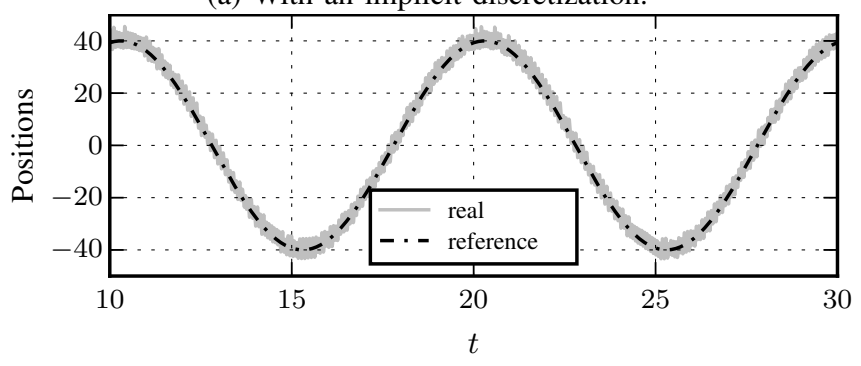

(b) With an explicit discretization.

Fig. 3: Real and desired position trajectories with $h=10 \mathrm{~ms}$ and $G=10^{5}$.

form of a boundary layer around the reference trajectory. The chattering has been drastically reduced with the use of an implicit controller. Turning our attention to the control input,

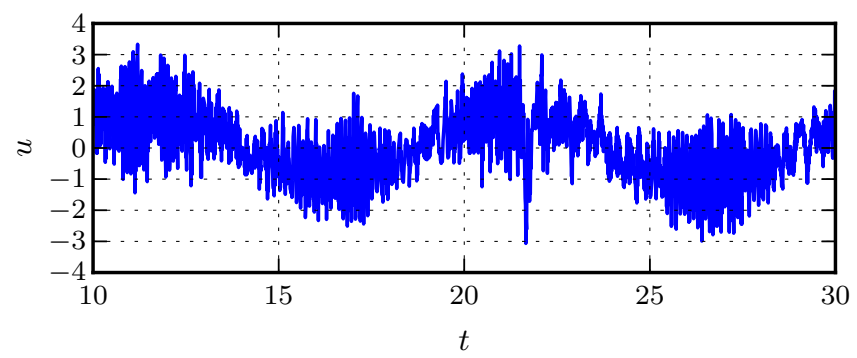

(a) With an implicit discretization.

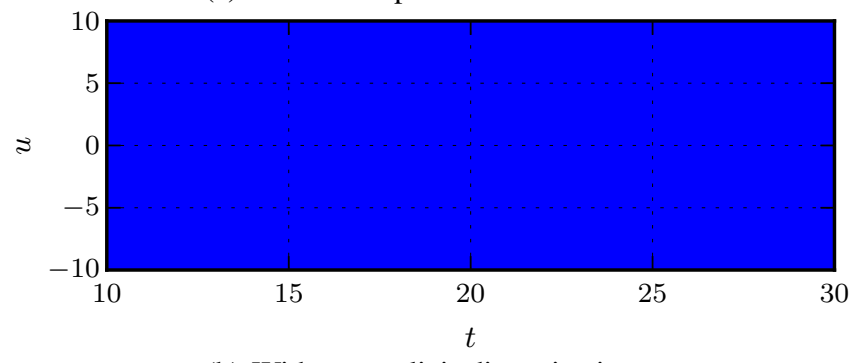

(b) With an explicit discretization.

Fig. 4: Evolution of the control input $u$ for both implicit and explicit discretization with $h=10 \mathrm{~ms}$ and $G=10^{5}$.

Fig. $4 \mathrm{a}$ and $4 \mathrm{~b}$ illustrate the evolution of this quantity in the implicit and explicit cases. In the first case, the control values are in the range $[-3,3.3]$, which is well inside the constraints $u \in[-10,10]$. Although the control is affected by the noise from the measurements, there is an underlining periodical signal, which is also witnessed on simulation results. The root cause is likely to be the approximations done to get the discrete-time model in (11). It is difficult to analyze the data on Fig. $4 \mathrm{~b}$ since the control input is switching at a very high frequency between the 2 extremal values -10 and 10 , sign of a chattering input. It is pretty clear that the main source of chattering is the explicit discretization of the controller. We analyze further the chattering in the second part of this section.

Having exposed the superiority of the implicit discretization with respect to the explicit one, let us further present the good performances that it yields. Firstly it is possible to

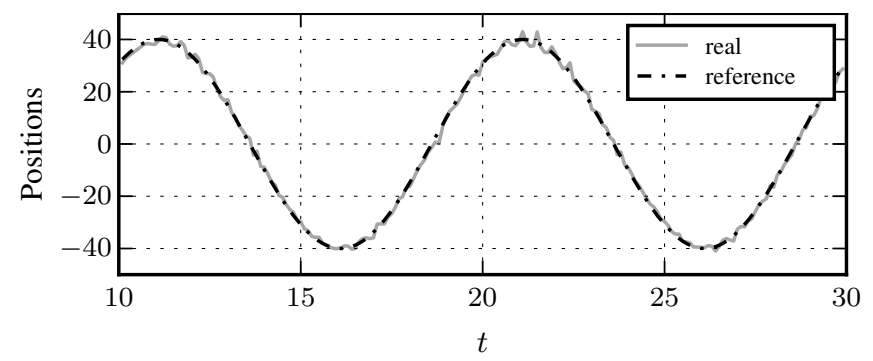

Fig. 5: Real and desired positions with an implicit discretization, $h=100 \mathrm{~ms}$ and $G=10^{5}$.

increase the sampling period while keeping a good tracking and a practically stable system. Fig. 5 illustrates this fact: even with a sampling period of $100 \mathrm{~ms}$, the tracking takes place, although with degraded performances compared to the one in Fig. 3a. However the precision is still better than with an explicit controller with a sampling period one order of magnitude smaller as shown in Fig. 2 and 3b. Another very

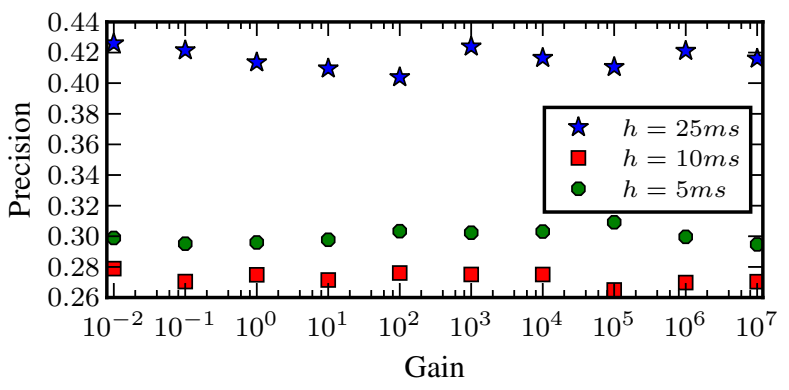

Fig. 6: Precision versus gain $(G$ in (9)).

nice feature of the implicit discretization is the fact that the control input value is computed as a selection, as mentioned earlier. One implication is that to be robust, the gain needs to be large enough with respect to the perturbation (see (3)) but a further increase in the gain does not harm the performances. This is illustrated by Fig. 6, where the precision $\bar{e}$ is plotted versus the gain $G$ for 3 sampling periods. For each value of the latter, we changed the gain from $10^{-2}$ to $10^{7}$, each time multiplied by 10 . In each case, the precision varies only by less than $5 \%$, with is solely due to the noise in the plant. The random evolution, with respect to the gain, in Fig. 6 further supports this claim. The insensitivity of the discontinuous controller w.r.t. the gain variations, has also been verified for the ECB-SMC controller in [13]. Let us switch focus on the chattering in the rest of the section.

\section{B. Input and output chattering}

We propose to characterize the chattering of a variable by the variation of the associated signal. The variation of a 
real-valued function $f(\cdot)$ is defined as

$$
\operatorname{Var}_{t_{0}}^{T}(f):=\sum_{k}\left|f\left(t_{k}\right)-f\left(t_{k-1}\right)\right|,
$$

with $k \in \mathbb{N}^{*}$ such that $t_{k} \in\left(t_{0}, T\right]$. Though this quantity is not commonly used in Control Engineering, it provides a nice characterization of the chattering on either the control input or the sliding variable. We pay attention to both input and output chattering, since the first one contributes to the second and it can also induce rapid wear of actuators, especially if they are mechanical ones. Furthermore, it may also be linked to the energy consumption of the actuator. As before,

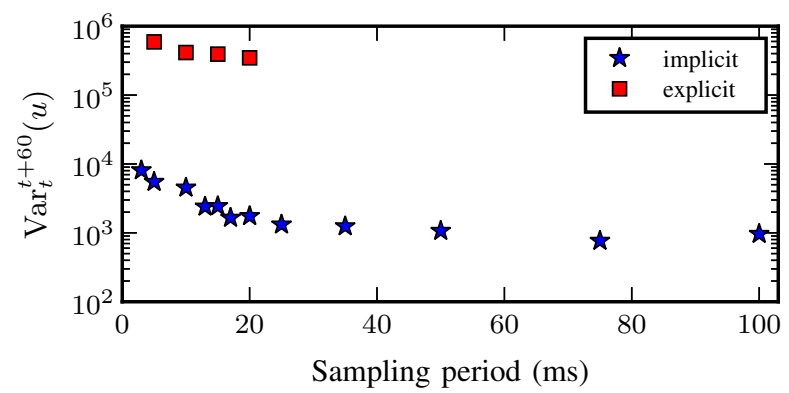

Fig. 7: Evolution of the control input variation with respect to the sampling time for both implicit and explicit discretizations. The gain used in every capture was $G=10^{5}$.

we present the evolution of the control input chattering with respect to the sampling period for both implicit and explicit controllers. From Fig. 7, we can infer that the trend in both cases is a decrease of the variation with an increase in the sampling period. Again the implicit controller performs much better, having a control input variation 2 orders of magnitude smaller than the explicit one. This reduced chattering can also be assessed on site with a huge reduction of the noise made by the actuators ${ }^{2}$.

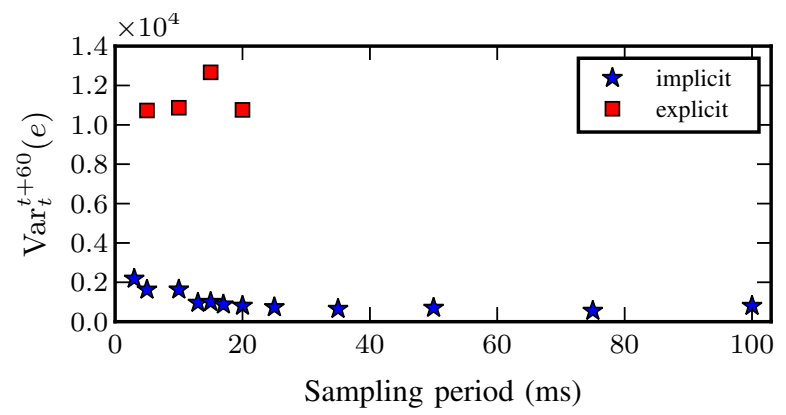

Fig. 8: Evolution of the error variation with respect to the sampling time for both implicit and explicit discretizations. The gain used in every capture was $G=10^{5}$.

Moving on to the output chattering, the same conclusion follows: the implicit method performs better than the explicit one, this time by an order of magnitude (see Fig. 8). This means that the output chattering is notably reduced. Indeed a bang-bang type control input, like the one the explicit discretization yields, tends to change the sign of the sliding

\footnotetext{
${ }^{2}$ The reader is invited to watch the videos at http://bipop. inrialpes.fr/people/huber/
}

variable very frequently. This leads to a large variation of the error, with respect to the variation with an implicit controller. At the same time, this behaviour does not yield a better tracking, as illustrated by Fig. 2.

\section{CONCLUSion}

In this note we present the results of an experimental study of two discrete-time twisting controllers: the implicit and the explicit one. Extensive experiments were conducted in the context of a position tracking problem. The analysis of the data reveals that on this electropneumatic setup, the implicit twisting controller outperforms the explicit one on 3 criteria: the tracking error and both the input and output chattering. Despite the complexity of the control loop arising from the high relative degree, meaningful illustrations of theoretical results are provided, like the insensitivity with respect to an increase in the gain, once the latter is large enough. The implicit discretization allows to drastically reduce both the output and the input chattering, without modifying the controller structure compared to its continuous-time version. Video recordings of the experiments can be found online ${ }^{2}$.

\section{REFERENCES}

[1] A. Levant, "Sliding order and sliding accuracy in sliding mode control," International Journal of Control, vol. 58, no. 6, pp. 1247$1263,1993$.

[2] M. Taleb, A. Levant, and F. Plestan, "Pneumatic actuator control: Solution based on adaptive twisting and experimentation," Control Engineering Practice, vol. 21, no. 5, pp. 727-736, 2013.

[3] V. Acary and B. Brogliato, "Implicit Euler numerical scheme and chattering-free implementation of sliding mode systems," Systems \& Control Letters, vol. 59, no. 5, pp. 284-293, 2010.

[4] V. Acary, B. Brogliato, and Y. Orlov, "Chattering-free digital slidingmode control with state observer and disturbance rejection," Automatic Control, IEEE Transactions on, vol. 57, no. 5, pp. 1087-1101, 2012.

[5] O. Huber, V. Acary, and B. Brogliato, "Analysis of explicit and implicit discrete-time equivalent-control based sliding mode controllers," INRIA, Rapport de Recherche RR-8383, 2013. [Online]. Available: http://hal.inria.fr/hal-00875209

[6] Y. Orlov, "Finite time stability and robust control synthesis of uncertain switched systems," SIAM Journal on Control and Optimization, vol. 43, no. 4, pp. 1253-1271, 2005.

[7] A. Polyakov and A. Poznyak, "Lyapunov function design for finitetime convergence analysis: "twisting" controller for second-order sliding mode realization," Automatica, vol. 45, no. 2, pp. 444-448, 2009.

[8] F. Facchinei and J.-S. Pang, Finite-Dimensional Variational Inequalities and Complementarity Problems, Springer Series in Operations Research. Springer, 2003.

[9] M. Cao and M. C. Ferris, "A pivotal method for affine variational inequalities," Mathematics of Operations Research, vol. 21, no. 1, pp. 44-64, February 1996.

[10] V. Acary and F. Pérignon, "An introduction to Siconos," INRIA, Rapport Technique RT-0340, 2007. [Online]. Available: http://hal.inria.fr/inria-00162911

[11] Y. Shtessel, M. Taleb, and F. Plestan, "A novel adaptive-gain supertwisting sliding mode controller: Methodology and application," Automatica, vol. 48, no. 5, pp. 759-769, 2012.

[12] S. Sesmat and S. Scarvarda, "Static characteristics of a three way electropneumatic servovalve," in Proceedings of the 12th Conference on Fluid Power Technology, Aachen, Germany, 1996, pp. 643-645.

[13] B. Wang, B. Brogliato, V. Acary, A. Boubakir, and F. Plestan, "Experimental comparisons between implicit and explicit implementations of discrete-time sliding mode controllers: Towards chattering suppression in output and input signals," in Variable Structure Systems (VSS), 2014 13th International Workshop on, June 2014, pp. 1-6.

[14] A. Levant, "Robust exact differentiation via sliding mode technique," Automatica, vol. 34, no. 3, pp. 379-384, 1998. 